\title{
Time Series Analysis of Mean Monthly Temperature in the Far Western Ethiopia, Assosa District
}

Tigabu Hailu Kassa ( $\nabla$ tigabu.hailu2004@gmail.com )

Samara University https://orcid.org/0000-0002-1253-7468

Shewayiref Geremew Gebremichael

Debre Tabor University

\section{Research}

Keywords: Time series analysis, Time series, Autoregressive moving average model, Global warming, Stationary process, Autocorrelation, Climate change, Mean squared error, Autoregressive model, Regression analysis

Posted Date: October 23rd, 2020

DOl: https://doi.org/10.21203/rs.3.rs-94148/v1

License: (9) This work is licensed under a Creative Commons Attribution 4.0 International License. Read Full License 
TIME SERIES ANALYSIS OF MEAN MONTHLY TEMPERATURE IN THE FAR WESTERN ETHIOPIA, ASSOSA DISTRICT

Tigabu Hailu Kassa ${ }^{1 *}$ and Shewayiref Geremew Gebremichael ${ }^{2}$

${ }^{1}$ Department of Statistics, Samara University, Ethiopia

${ }^{2}$ Department of Statistics, Debre Tabor University, Ethiopia

*Corresponding Author

E-mail: tigabu.hailu2004@gmail.com 


\begin{abstract}
Background

This study investigated the mean monthly temperature pattern of the Assosa district, Western Ethiopia. The objective of this study was to analyze the mean monthly temperature patterns in the Assosa district for the period from January 2012 to December 2016 based on data from meteorological stations in the Assosa district.
\end{abstract}

\title{
Methods
}

Descriptive statistics and univariate Box-Jenkins methodology to build the seasonal ARIMA model were used.

\section{Results}

The results showed that the mean annual temperature of Assosa was 28.025 degree Celsius. The original series was found to be seasonally non-stationary, as indicated by the ACF plot of the series. After using first-order seasonal differencing, the series was found to be stationary. A time-series model for the Assosa station was adjusted, processed, diagnostically checked, and finally, an ARIMA (3.0.1) model is established and this model is used to forecast one-year mean monthly temperature values.

\section{Conclusion}

The forecasted mean temperature values showed a similar pattern to previous recordings.

Keywords: - Time series analysis, Time series, Autoregressive moving average model, Global warming, Stationary process, Autocorrelation, Climate change, Mean squared error, Autoregressive model, Regression analysis 


\section{Background}

Global warming is considered a major threat to life on our planet. Observations show that the global mean temperature at the earth's surface has substantially increased over the twentieth century (IPCC, 2007). This global warming and its multifaceted impacts are affecting the whole world in various forms. Several scientific studies have suggested that developing countries in particular are suffering from the burden of ever-changing climatic conditions (UN-OHRLLS, 2009). Many low-income countries are located in tropical, subtropical regions, or semi-arid zones, which are particularly vulnerable to shifting weather patterns and rising temperatures (Joachim, 2008). Widespread research findings have revealed that climate variability and change have significant impacts on global and regional food production systems, particularly on the performance of common staple food crops in the tropical subhumid climatic zone (UNOHRLLS, 2009). Climate variability and change have been implicated to have significant impacts on global and regional food production, particularly the common staple food crop performance in tropical subhumid climatic zones. For example, the most food-insecure regions and most climate change vulnerable regions in Ethiopia are those that experience both the lowest and most variable rainfall patterns (UN-OHRLLS, 2009). Many African countries are vulnerable to climate change because their economies largely depend on climate-sensitive agricultural production systems (Temesgen, 2000). This is particularly true in low-income countries like Ethiopia, where adaptive capacity is low.

Ethiopia is characterized by diverse topographic features that have led to the existence of a range of agro-climatic zones, each with distinctly variant climatic conditions, which in turn have resulted in the evolution of a wide variety of fauna and flora and agricultural production systems. Among these agro-climatic zones, the lowland ('kola') that receives the lowest and most erratic rainfall rates, notably the Central Rift Valley (CRV) region, experiences frequent natural hazards such as sudden flooding, recurrent droughts, and chronic water stress, which are aggravated by climate change and its variability. Climate variability of erratic rainfall and its uneven sequential and spatial distribution creates frequent flooding and drought in these areas (Lai et al., 1998).

The climate in Ethiopia varies tremendously depending on elevation. Ethiopia is already experiencing a change in its climate. Between 1951 and 2006, the annual minimum temperature in Ethiopia has increased by about $0.37^{\circ} \mathrm{C}$ every decade. The UNDP Climate Change profile for 
Ethiopia also shows that the mean annual temperature has increased by $1.3^{\circ} \mathrm{C}$ between 1960 and 2006, at an average rate of $0.28^{\circ} \mathrm{C}$ per decade (McSweeney. 2010). The temperature increase was most rapid from July to September $\left(0.32^{\circ} \mathrm{C}\right.$ per decade). Reportedly, the average number of hot days per year has increased by 73 (an additional 20\% of days) and the number of hot nights has increased by 137 (an additional 37.5\% of nights) between 1960 and 2006. The rate of increase was highest in June, July, and August. Over the same period, the average number of cold days and nights has decreased by 21 (5.8\% of days) and 41 (11.2\% of nights), respectively. These reductions have mainly occurred from September to November (McSweeney,2010).

Climate change is real and happening now. The average global surface temperature has warmed by $0.8^{\circ} \mathrm{C}$ in the past century and $0.6^{\circ} \mathrm{C}$ in the past three decades (Hansen et al., 2006). The effects of climate change and variabilities, such as rising temperature and changes in precipitation, are undeniably clear with impacts already affecting ecosystems, biodiversity, and people (IPCC, 2001).

In contemporary thinking, the term climate change has become a core issue in various developmental, environmental, social, and political forums at the grassroots, national, regional, and international levels (Akililu and Alebachew, 2009).

However, climate change is typically discussed in global terms; its effects vary quite dramatically among different regions of the earth, but what one does know for certain is that this warming trend is expected to continue, increasing at dramatic rates. To better understand the impact of climate change has on cities, one must look at the scientific evidence of global and regional climate change, its primary causes, and recorded and projected effects (IPCC, 2001).

The Intergovernmental Panel on Climate Change (IPCC) in 2007 concluded that warming of the climate system was unequivocal and very likely (more than a 90\% chance) caused by humans (Ares et al., 2009). The study also concluded that global emissions would have to peak in 2015 and reduced by $25-40 \%$ in 2020 and by $50-85 \%$ in 2050 to have a $50 \%$ chance of limiting global temperature increase to $2^{\circ} \mathrm{C}$.

Water vapor and other "greenhouse gases" such as carbon dioxide, methane, and CFCs cause the greenhouse effect by trapping radiant heat emitted at infrared (long) wavelengths (as opposed to shorter, solar wavelengths which can pass through the atmospheric gases) from the earth's surface and reradiating it back to the earth's surface and trapped energy effectively, creates an 
enclosure around the earth's atmosphere similar to a greenhouse, which not only traps heat but also restricts air circulation that would otherwise cause cooling (Botkin and Keller, 2000).

Natural processes also lead to climate variability and changes that occur on all time scales across the Earth. Natural climate variability on multiple time scales (ranging from interannual, multidecadal, and longer geologic-time scales) is a major obstacle to the reliable characterization of global climate change resulting from human activities (Ghil, 2002).

Many scientists believe that natural global cooling mechanisms worked to offset warming. With the release of the IPCC's Third Assessment Report in 2001, however, came new evidence that climate change over the last 50 years was, indeed, attributable to humans, mostly from burning fossil fuels that emit more carbon dioxide into the atmosphere than it should contain to achieve climate equilibrium ( 5.4 billion metric tons annually) and two additional human causes of global warming include deforestation, which adds another 1.6 billion metric tons, and emission of other greenhouse gases (methane, CFCs, ozone, nitrous oxides) that also contribute to the greenhouse effect (Botkin and Keller,2000).

Climate change is emerging as one of the main challenges humankind will have to face for many years to come. It could become a major risk to world food security, as it has a strong effect on food production, access, and distribution. Global atmospheric concentrations of anthropogenic greenhouse gases (methane, carbon dioxide, and nitrous oxide) have increased markedly since 1950. Due to this increase, the global surface temperature has also been observed to have been increasing (IPCC, 2007). Therefore, this study is undertaken to analyze the mean monthly temperature of the Assosa district and to forecast the mean monthly temperature of the area. 


\section{Materials and Methods}

\subsection{Data Source}

Monthly time series data on mean monthly temperature for the period January 2012 to December 2016, collected by the National Meteorological Agency of the Assosa station, Ethiopia, were used in the study.

\subsection{Statistical Analysis}

The statistical analysis and model that is used are time series analysis. Time series is broadly defined as any series of measurements taken at different times. There are four components of the time series. These are the trend, seasonal, cyclical, and irregular components.

\subsubsection{Data Analysis in Time Series}

\section{Test of stationary}

Before fitting a particular model to time series data, the series must be made stationary. Stationarity occurs in a time series when there is no systematic change in mean (no trend) and if there is no change in the variance of the series. To test for stationarity of a Series several procedures have been developed. We use the following four relatively simple techniques:

Time plot: The first step in the analysis of time series is usually to plot the data and obtain simple descriptive measures of the main property of the series via a visual inspection of the time series plot. If there is a trend either in the mean level or the variance of the series, then this is the sign of non-stationary.

Correlogram Test: One way to characterize a series to its dependence over time is to plot its sample autocorrelation function (SACF). The sample partial autocorrelation function, denoted by SPACF, is similar to the SACF and can be described as the correlation between $y_{t}$ and $y_{t-s}$ (observations of the time series recorded at two moments in time s time units apart) after controlling for the common linear effects of the intermediate lags. Both functions are used in Box-Jenkins modeling as a correlogram to reveal important information regarding the order of the autoregressive (AR) and moving average (MA) factors present in the generating process of the given time series as well as to assess stationarity. Enders (1995) expresses that the inspection of SACF serves as a rough indicator of whether non-stationarity is present in a series. Wei (1990) stated that if the sample ACF decays very slowly, this indicates that a difference is 
needed. The tests of stationarity described above make use of subjective visual inspection of data; formal tests were developed to help determine stationarity.

Unit Root Test: The Unit Root test is frequently employed for testing stationarity. The test first poses the null hypothesis that the given time series has a unit root, which means that the time series is non-stationary, and tests if the null hypothesis is to be statistically rejected in favor of the alternative hypothesis that the given time series is stationary. To detect whether a given series is non-stationary, let us assume that the relationship between the current value (in time $\mathrm{t}$ ) and last value (in time t-1) in the time series is given as follows.

$$
Y_{t}=\alpha Y_{t-1}+\varepsilon_{t}
$$

This model is a first-order autoregressive process. The time series $Y_{t}$ converges, as $t \rightarrow \infty$, to a stationary time series if $|\alpha|<1$. If $|\alpha|=1$ or $>1$, the series $Y_{t}$ is not stationary and the variance of $Y_{t}$ is time-dependent. In other words, the series has a unit root.

The null hypothesis $\left(H_{o}\right)$ is that there is a unit root, that is, $\alpha=1$, and the alternative $\left(H_{1}\right)$ is stationary, which is equivalent to $\alpha<1$, assuming, as seems reasonable, that $\alpha>-1$. Model (1) is equivalent to $\Delta Y_{t}=(\alpha-1) Y_{t-1}+\varepsilon_{t}$ since, $\Delta y_{t}=y_{t}-y_{t-1}(\mathrm{D}$. Ruppert, 2011)

$$
\Delta Y_{t}=\gamma Y_{t-1}+\varepsilon_{t}
$$

Where $\gamma=\alpha-1$

The Unit Root test subsequently tests the following one-sided hypothesis.

$$
\begin{gathered}
H_{o}: \alpha=1 \text { (The time series data are not stationary), } \\
H_{o}: \gamma=0 \text { (the data needs to be differenced to make it stationary) }
\end{gathered}
$$

$H_{o}: \gamma=0$ (time series is non-stationary),

$H_{o}: \gamma=0$ (the series has a unit root)

$H_{o}: \alpha<1$ (the time series data are stationary)

$H_{1}: \gamma<0$ (the data is stationary and does not need to be differenced) 


\section{Augmented Dickey-Fuller (ADF) Test}

By including lags of order $p$, the ADF formulation allows for higher-order autoregressive processes. This means that the lag length $p$ has to be determined when applying the test. Although several ways of choosing $\mathrm{p}$ have been proposed, they are all arbitrary and are thus not presented here. Instead, the following two simple rules of thumb are suggested. First, the frequency of the data can be used to decide. Therefore, for example, if the data are monthly, we use 12 lags if the data are quarterly, use 4 lags, and so on. An alternative approach is to examine information criteria such as the Akaike information criterion and Bayesian information criterion.

The Dickey-Fuller test regresses $\Delta Y_{t}$ on $Y_{t-1}$ and tests $H_{o}$. Because $Y_{t-1}$ is non-stationary under $H_{o}$, the t-statistic for $\gamma$ has a nonstandard distribution so special tables need to be developed to compute p-values.

The augmented Dickey-Fuller test expands model (2) by adding a time trend and lagged values of $\Delta Y_{t}$. Typically, the time trend is linear, and the expanded model is shown in equation (3).

The testing procedure for the ADF test is applied to the model:

$$
\begin{array}{r}
\Delta y_{t}=\beta_{o}+\beta_{1} \mathrm{t}+\gamma y_{t-1}+\phi_{1} \Delta y_{t-1}+\phi_{2} \Delta y_{t-2}+\cdots+\phi_{p} \Delta y_{t-p}+\varepsilon_{i} \\
\Delta y_{t}=\beta_{o}+\beta_{1} \mathrm{t}+\gamma y_{t-1}+\sum_{j=1}^{p} \phi_{j} \Delta y_{t-j}+\varepsilon_{i}
\end{array}
$$

Where, $\beta_{o}$ is a constant, $\beta_{1}$ is the coefficient on a time trend, and " $\mathrm{p}$ " the lag order of the autoregressive process.

Imposing the constraints $\beta_{o}=0$ and $\beta_{1}=0$ corresponds to modeling a random walk and using the constraint $\beta_{1}=0$ corresponds to modeling a random walk with a drift. The test was then carried out as

$$
\begin{gathered}
H_{o}: \gamma=0 \text { Nonstationary } \\
H_{1}: \gamma<0 \text { Stationary }
\end{gathered}
$$

The hypotheses are still $H_{o}: \gamma=0$ and $H_{1}: \gamma<0$. There are several methods for selecting 'p'. The adf.test function in R software has a default value of "p" equal to trunk((length $\left.(x)-1)^{\wedge}(1 / 3)\right)$, where " $x$ " is the input series ( $y_{t}$ in our notation) (D. Ruppert, 2011). 
Test statistic is given by:

$D F_{T}=\frac{\widehat{\gamma}}{S E(\widehat{\gamma})}$ is computed and compared to the critical value for the Dickey-Fuller Test. If the test statistic is less than the critical value, then the null hypothesis of $\gamma=0$ is rejected and no unit root is presented.

\section{Phillips-Perron (PP) Test}

Phillips and Perron (1988) propose an alternative (non-parametric) method of controlling for serial correlation when testing for stationarity. The PP test estimates the non-augmented DFtest and modifies the $\mathrm{t}$ - ratio of the $\alpha$ coefficient so that serial correlation does not affect the asymptotic distribution of the test statistic. The testing procedure for the PP test was applied to the model:

$$
y_{t}=c+\delta t+\alpha y_{t-1}+\varepsilon_{t}
$$

Where, with drift coefficient $c$, deterministic trend coefficient $\delta$, and $\operatorname{AR}(1)$ coefficient $\alpha<1$.

The null hypothesis restricts $a=1$. Variants of the test, appropriate for series with different growth characteristics, restrict the drift and deterministic trend coefficients, $c$ and $\delta$, respectively, to be 0 . The tests use modified Dickey-Fuller statistics to account for serial correlations in the innovations process $\varepsilon_{t}$.

The PP test is based on the test statistic:

$$
\hat{t}=t_{\alpha}\left(\frac{\gamma_{o}}{f_{o}}\right)^{1 / 2}-\frac{T\left(f_{o}-\gamma_{o}\right)}{2\left(f_{o}{ }^{1 / 2}\right) * s}(\operatorname{se}(\hat{a}))
$$

Where $\hat{c}$ is the estimate of $\mathrm{c}, t_{\alpha}$ is the $\mathrm{t}$-ratio of $\mathrm{c}$, se $(\hat{c})$ is the coefficient standard error and $\mathrm{s}$ is the standard error of the test regression. Besides, yo is a consistent estimate of the error variance; the remaining term, $\mathrm{f}_{\mathrm{o}}$, is an estimator of the residual spectrum at frequency zero.

\subsubsection{Transformations to achieve stationarity}

Transformation is the process of changing a non-stationary time series into a stationary time series. Non-stationary series can be transformed into stationary series using the following methods. 
1. Differencing the data. That is, given the series $Y_{t}$, we create the new series

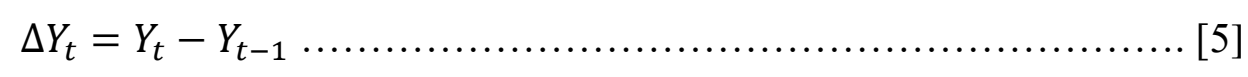

The differenced data contain one pointless than the original data. Although you can differentiate the data more than once, the first difference is usually sufficient to achieve stationarity.

2. If the data are seasonally non-stationary, regular differencing cannot achieve the stationarity of the series. Therefore, we can use seasonal differencing to achieve the stationarity of the series.

3. If the data contain a trend, we can fit some type of curve to the data and then model the residuals from that fit. Because the purpose of the fit is to simply remove long-term trends, a simple fit, such as a straight line, is typically used.

4. For non-constant variance, taking the logarithm or square root of the series may stabilize the variance. For negative data, you can add a suitable constant to make the entire data positive before applying the transformation. This constant can then be subtracted from the model to obtain predicted (i.e., the fitted) values and forecasts for future points.

\subsection{Time series model}

\subsubsection{White Noise (pure random) process}

A time series is called white noise if $\left\{y_{t}\right\}$ is a sequence of independently and identically distributed random variables with mean zero and constant variance. The series $Y_{t}=\varepsilon_{t}$ describes a purely random process with

$$
\begin{gathered}
E\left(\varepsilon_{t}\right)=0 \\
\operatorname{Var}\left(\varepsilon_{t}\right)=\sigma^{2}
\end{gathered}
$$

$E\left(\varepsilon_{t} \varepsilon_{s}\right)=0 \forall_{t \neq s}$, and also $\varepsilon_{t}$ is normally distributed. This assumption is expressed as follows:

$$
\varepsilon_{t} \sim \underline{\text { i.i.d }} N\left(0, \sigma^{2}\right)
$$

where i.i.d stands for independently and identically distributed random variable.

\subsubsection{Autoregressive process of order $p, A R(p)$}

Assume the error term is purely random with mean zero and variance $\sigma^{2}$, then the process $y_{t}$ is said to be an autoregressive process of order $\mathrm{p}$ if: 


$$
y_{t}=\alpha_{1} y_{t-1}+\alpha_{2} y_{t-2}+\cdots+\alpha_{p} y_{t-p}+\varepsilon_{t}
$$

where, $y_{t}$ is stationary series, $\alpha_{1}, \alpha_{2}, \ldots, \alpha_{p}$ is parameters of $\operatorname{AR}\left(\alpha_{p} \neq 0\right)$

\section{Using Operators}

The backward shift operator, $\mathrm{B}$, is defined such that $B y_{t}=y_{t-1}$. In general, this implies that $B^{j} y_{t}=y_{t-j}$

Let $B^{2} y_{t}$

$$
B^{2} y_{t}=B\left(B y_{t}\right)=B y_{t-1}=y_{t-2}
$$

The backward difference operator, $\nabla$, may be defined as $\nabla y_{t}=y_{t}-y_{t-1}$

$$
\begin{aligned}
& \begin{array}{l}
\nabla y_{t} \\
\nabla y_{t}=y_{t}-y_{t-1}=y_{t}-B y_{t}=(1-B) y_{t} \text { since, } B y_{t}=y_{t-1} \text { and therefore, } \nabla=1-\mathrm{B} \\
\nabla y_{t}=y_{t}-y_{t-1}
\end{array} \\
& \begin{array}{c}
\nabla^{2} y_{t} \\
\nabla^{2} y_{t}=\nabla \nabla y_{t}=(1-B)(1-B) y_{t} \text { since, } \nabla=(1-B) \\
\nabla^{2} y_{t}=\nabla \nabla y_{t}=(1-B)(1-B) y_{t}=\left(1-2 B+B^{2}\right) y_{t} \\
\nabla^{3} y_{t} \\
\nabla^{3} y_{t}=\nabla \nabla \nabla y_{t}=(1-B)(1-B)(1-B) y_{t} \text { as usual } \nabla=1-\mathrm{B} \\
\nabla^{3} y_{t}=\nabla \nabla \nabla y_{t}=(1-B)\left(1-2 B+B^{2}\right) y_{t}=\left(1-2 B+B^{2}-B+2 B^{2}-B^{3}\right) y_{t} \\
\nabla^{3} y_{t}=\left(1-3 B+3 B^{2}-B^{3}\right) y_{t}=\left(1-3 B+3 B^{2}-3 B^{3}\right) y_{t}
\end{array}
\end{aligned}
$$

Shortly,

$$
\begin{gathered}
B^{1} y_{t}=B y_{t}=y_{t-1} \\
B^{2} y_{t}=B B y_{t}=B y_{t-1}=y_{t-2} \\
B^{3} y_{t}=B^{2} B y_{t}=B B y_{t-1}=B y_{t-2}=y_{t-3} . \\
\text { with similar fashion } \\
B^{p} y_{t}=y_{t-p} . \\
y_{t}=\alpha_{1} y_{t-1}+\alpha_{2} y_{t-2}+\cdots+\alpha_{p} y_{t-p}+\varepsilon_{t} \text { can be rewrite as }
\end{gathered}
$$




$$
\begin{gathered}
y_{t}-\alpha_{1} y_{t-1}-\alpha_{2} y_{t-2}-\cdots-\alpha_{p} y_{t-p}=\varepsilon_{t} \\
y_{t}-\alpha_{1} B y_{t}-\alpha_{2} B^{2} y_{t}-\alpha_{3} B^{3} y_{t} \cdots-\alpha_{p} B^{p} y_{t-p}=\varepsilon_{t}
\end{gathered}
$$

From the above concepts the model in lag operators takes the following form:

$$
\left(1-\alpha_{1} B-\alpha_{2} B^{2}-\alpha_{p} B^{p}\right) y_{t}=\varepsilon_{t}
$$

Where, the lag (backshift) operator B is defined as:

$$
B^{p} y_{t}=y_{t-p}
$$

Where, $p=0,1,2, \ldots$

\subsubsection{Moving Average Model of Order q, MA (q)}

Suppose that $\varepsilon_{\mathrm{t}}$ is a purely random process with mean zero and variance $\sigma^{2}$, then a process $y_{t}$ defined by

$$
y_{t}=\varepsilon_{t}+\beta_{1} \varepsilon_{t-1}+\beta_{2} \varepsilon_{t-2}+\cdots+\beta_{q} \varepsilon_{t-q}
$$

where, $\beta_{1}, \beta_{2}, \ldots, \beta_{p}$ are parameters of MA.

$M A(q)$ Models immediately define stationary, and every MA process of finite order is stationary (Diebold et al., 2006). To preserve a unique representation, the requirement is usually imposed such that all roots of $1+\beta_{1} B+\beta_{2} B^{2}+\beta_{q} B^{p}=0$ are greater than one in absolute value. If all the roots lie outside the unit circle MA (q) can be represented as AR ( $\infty)$, thus we expect the opposite patterns for MA processes. The PACF dampens exponentially. ACF was used to identify the order of the MA process.

\subsubsection{Autoregressive Moving Average, ARMA (p, q)}

We now proceed with the general development of autoregressive, moving average, and mixed autoregressive moving average (ARMA) models for stationary time series. In most cases, it is best to develop a mixed autoregressive moving average model when building a stochastic model to represent a stationary time series. The order of an ARMA model is expressed in terms of both $p$ and $q$. The model parameters relate to what happens in period $t$ to both the past values and the random errors that occurred in past periods.

It is given by the following the general equation: 


$$
y_{t}=\alpha_{1} y_{t-1}+\cdots+\alpha_{p} y_{t-p}+\varepsilon_{t}+\beta_{1} \varepsilon_{t-1}+\cdots+\beta_{q} \varepsilon_{t-q}
$$

Equation (8) of the time series model can be simplified by a backward shift operator B to obtain (9):

$$
\begin{aligned}
& y_{t}-\alpha_{1} y_{t-1}-\cdots-\alpha_{p} y_{t-p}=\varepsilon_{t}+\beta_{1} \varepsilon_{t-1}+\cdots+\beta_{q} \varepsilon_{t-q} \\
& y_{t}-\alpha_{1} B y_{t}-\alpha_{2} B^{2} y_{2}-\cdots-\alpha_{p} B^{p} y_{t}=\varepsilon_{t}+\beta_{1} \varepsilon_{t-1}+\cdots+\beta_{q} \varepsilon_{t-q} \\
& \left(y_{t}-\alpha_{1} B y_{t}-\alpha_{2} B^{2} y_{t}-\cdots-\alpha_{p} B^{p} y_{t}\right)=\varepsilon_{t}+\beta_{1} B \varepsilon_{t}+\beta_{2} B^{2} \varepsilon_{t}+\cdots+\beta_{q} B^{p} \varepsilon_{t} \\
& \left(1-\alpha_{1} B-\alpha_{2} B^{2}-\cdots-\alpha_{p} B^{p}\right) y_{t}=\left(\varepsilon_{t}+\beta_{1} B \varepsilon_{t}+\beta_{2} B^{2} \varepsilon_{t}+\cdots+\beta_{q} B^{p} \varepsilon_{t}\right) \\
& \left(1-\alpha_{1} B-\alpha_{2} B^{2}-\cdots-\alpha_{p} B^{p}\right) y_{t}=\left(1+\beta_{1} B+\beta_{2} B^{2}+\cdots+\beta_{q} B^{p}\right) \varepsilon_{t} \\
& \alpha(B) y_{t}=\beta(B) \varepsilon_{t}
\end{aligned}
$$

Where $\varepsilon_{t}$ is White Noise process and $\alpha(B)=1-\alpha_{1} B-\alpha_{2} B^{2}-\cdots-\alpha_{p} B^{p}$ and $\beta(B)=1+$ $\beta_{1} B+\beta_{2} B^{2}+\cdots+\beta_{q} B^{p}$

The ARMA model is stable, i.e., it has a stationary "solution" -if all roots of $\boldsymbol{\alpha}(\boldsymbol{B})=\mathbf{0}$ are larger than one in absolute value. The representation is unique if all roots of $\boldsymbol{\alpha}(\boldsymbol{B})=\mathbf{0}$ lie outside the unit circle and $\boldsymbol{\alpha}(\boldsymbol{B})$ and $\boldsymbol{\beta}(\boldsymbol{B})$ does not have common roots. Stable ARMA models always have an infinite order MA representation. To have ARMA $(p, q)$ model, both ACF and PACF should show a pattern of decaying to zero. The autocorrelation of an $\operatorname{ARMA}(p, q)$ process is determined at a greater lag of the AR $(p)$ part of the process as the effect of the MA part dies out. Thus, eventually, the ACF consists of mixed damped exponentials and sine terms. Similarly, the partial autocorrelation of an $\operatorname{ARMA}(p, q)$ process is determined at a greater lag of the MA $(q)$ part of the process. Thus, eventually, the partial autocorrelation function also consists of a mixture of damped exponentials and sine waves.

\subsubsection{Autoregressive integrated moving average ARIMA (p, d, q)}

Autoregressive integrated moving average (ARIMA) models are a specific subset of univariate modeling, in which a time series is expressed in terms of past values of itself (the autoregressive component) plus current and lagged values of a "white noise" error term (the moving average component). ARIMA models are univariate models that consist of an autoregressive polynomial, an order of integration (d), and a moving average polynomial. 
A process $\left(y_{t}\right)$ is said to be an autoregressive integrated moving average process, denoted by $\operatorname{ARIMA}(\mathrm{p}, \mathrm{d}, \mathrm{q})$ if it can be written as:

$$
\alpha(B) \nabla^{\mathrm{d}} y_{t}=\beta(B) \varepsilon_{t}
$$

Where $\nabla^{\mathrm{d}}=(1-\mathrm{B})^{\mathrm{d}}$

\subsubsection{Seasonal ARIMA (SARIMA) model}

In this section, we introduce several modifications made to the ARIMA model to account for seasonal and non-stationary behavior. Often, the dependence on the past tends to occur most strongly at multiples of some underlying seasonal lags.

For example, with monthly economic data, there is a strong yearly component occurring at lags that are multiples of $\mathrm{s}=12$, because of the strong connections of all activity to the calendar year. Data taken quarterly will exhibit the yearly repetitive period at $\mathrm{s}=4$ quarters. Seasonal ARIMA (SARIMA) is used when the time series exhibits seasonal variation. Nature phenomena such as temperature also have strong components corresponding to seasons. Hence, the natural variability of many physical, biological, and economic processes tends to match with seasonal fluctuations. Because of this, it is appropriate to introduce autoregressive and moving average polynomials that identify with the seasonal lags. The resulting pure seasonal autoregressive moving average model, say, ARMA (P, Q)s, then takes the form (Shumay and Stoffer, 2010):

$\Phi_{P}\left(B^{S}\right) x_{t}=\Theta_{\varrho}\left(B^{S}\right) w_{t}$

$$
\Phi_{P}\left(B^{s}\right) y_{t}=\Theta_{Q}\left(B^{s}\right) \varepsilon_{t}
$$

With the following definition of the operators

$$
\begin{gathered}
\Phi_{P}\left(B^{s}\right)=1-\Phi_{1} B^{s}-\Phi_{2} B^{2 s}-\cdots-\Phi_{p} B^{p s} \\
\text { and }
\end{gathered}
$$

$\Theta_{Q}\left(B^{s}\right)=1+\Theta_{1} B^{s}+\Theta_{2} B^{2 s}+\cdots+\Theta_{Q} B^{Q s}$ are the seasonal autoregressive operator and the seasonal moving average operator of orders $\mathrm{P}$ and Q, respectively, with seasonal period "s". 
Analogous to the properties of nonseasonal ARMA models, the pure seasonal ARMA $(P, Q) \mathrm{s}$ is causal only when the roots of $\Phi_{P}\left(B^{S}\right)$ lie outside the unit circle, and it is invertible only when the roots of $\Theta_{Q}\left(B^{S}\right)$ lie outside the unit circle (Shumay and Stoffer, 2010).

In general, we can combine the seasonal and non-seasonal operators into a multiplicative seasonal autoregressive moving average model, denoted by $\operatorname{ARMA}(p, q) \times(P, Q) s$, and write as the overall model as:

$$
\Phi_{P}\left(B^{s}\right) \alpha(\mathrm{B}) y_{t}=\Theta_{Q}\left(B^{s}\right) \beta(\mathrm{B}) \varepsilon_{t}
$$

A seasonal autoregressive notation $(P)$ and a seasonal moving average notation $(Q)$ form the multiplicative seasonal autoregressive integrated moving average model, denoted by SARIMA $(p, d, q)^{*}(P, D, Q) s$, of Box and Jenkins (1976) and is given by (Shumay and Stoffer, 2010):

$$
\Phi_{P}\left(B^{S}\right) \alpha(\mathrm{B}) \nabla_{s}^{D} \nabla^{d} y_{t}=\delta+\Theta_{Q}\left(B^{s}\right) \beta(\mathrm{B}) \varepsilon_{t}
$$

Where $\varepsilon_{t}$ is the usual Gaussian white noise process. The general model is denoted as SARIMA $(p, d, q) *(P, D, Q) s$. The ordinary autoregressive and moving average components are represented by polynomials $\alpha(\mathrm{B})$ and $\beta(\mathrm{B})$ of orders $\mathrm{p}$ and $\mathrm{q}$, respectively, and the seasonal autoregressive and moving average components by $\Phi_{P}\left(B^{S}\right)$ and $\Theta_{Q}\left(B^{S}\right)$ of orders $\mathrm{P}$ and $\mathrm{Q}$ and ordinary and seasonal difference components can be written as (Shumay and Stoffer, 2010):

$$
\nabla^{d}=(1-B)^{d} \text { and } \nabla_{s}^{D}=\left(1-B^{S}\right)^{D}
$$

\subsection{Building ARIMA Models}

To identify a perfect ARIMA model for a particular time-series data, Box and Jenkins (1976) proposed a methodology that consists of four phases: i) model identification, ii) estimation of model parameters, iii) diagnostic checking for the identified model, and iv) application of the model (i.e., forecasting).

\subsubsection{Model identification}

This step is the determination of the appropriate values of $\mathrm{p}, \mathrm{d}$, and $\mathrm{q}$. There are several identification methods have been proposed in the literature. The autocorrelations function (ACF) 
and partial autocorrelation function (PACF) are the two most useful tools in any attempt at time series model identification (Granger and Newbold, 1986).

Autocorrelation Function (ACF): The sample ACF $\left(r_{k}\right)$ measures the amount of linear dependence between observations in a time series that are separated by a lag k. To use the ACF in model identification, estimate $r_{k}$, and then plot $r_{k}$ series against lag $k$, which should be less than one-fourth of the series under study (Hipel et al., 1977). The autocorrelation of order $k$ is simply the correlation between $Y_{t}$ and $Y_{t-k}$, i.e.

$$
\rho_{k}=\frac{E\left\{\left(y_{t}-\bar{y}\right)\right\}\left\{\left(y_{t-1}-\bar{y}\right)\right\}}{E\left(y_{t}-\bar{y}\right)^{2}}
$$

In practice, one never knows the true autocorrelations and partial autocorrelations and at the identification stage, one has to rely on the sample autocorrelation and partial autocorrelation functions imitating the behavior of the corresponding parent quantities. True autocorrelations ( $\rho k)$ can be estimated by:

$$
\gamma_{k}=\frac{\frac{\sum_{t=k+1}^{n}\left(y_{t}-\bar{y}\right)\left(y_{t-k}-\bar{y}\right)}{n}}{\frac{\sum_{t=1}^{n}\left(y_{t}-\bar{y}\right)^{2}}{n}}
$$

\section{Partial Autocorrelation Function (PACF)}

The lag $\mathrm{k}$ partial autocorrelation is the partial regression coefficient, $\theta_{\mathrm{kk}}$ in the $\mathrm{k}^{\text {th }}$ order autoregression and is given by

$$
y_{t}=\theta_{k 1} y_{t-1}+\theta_{k 2} y_{t-2}+\theta_{k 3} y_{t-3}+\cdots+\theta_{k k} y_{t-k}+\varepsilon_{t}
$$

Table 2.1 Behavior of the ACF and PACF for ARMA (p, q) Models

\begin{tabular}{|l|l|l|l|}
\hline & AR(p) & MA(q) & ARMA(p,q) \\
\hline ACF & Tails off & Cuts off after lag q & Tails off \\
\hline PACF & Cuts off after lag $p$ & Tails off & Tails off \\
\hline
\end{tabular}

Table 2.2 Behavior of the ACF and PACF for SARMA Models 


\begin{tabular}{|l|l|l|l|}
\hline measures & AR(P)s & MA(Q)s & ARMA(P,Q) \\
\hline ACF & $\begin{array}{l}\text { Tails off at lags Ks, } \\
\mathrm{K}=1,2, \ldots\end{array}$ & Cuts off after lags Qs & Tails off at lags Ks \\
\hline PACF & Cuts off after lags Ps & Tails off at lags Ks & Tails off at lag Ps \\
\hline
\end{tabular}

where the values at non-seasonal lags $\mathrm{h} \neq \mathrm{Ks}$ for $\mathrm{K}=1,2 \ldots$ are zero. When the process is SARIMA $(0, d, q) x(0, D, Q) s$ model, $r_{k}$ truncates and is not significantly different from zero after lag $q+s Q$. If $r_{k}$ spikes out at lags that are multiples of $\mathrm{s}$, this implies the presence of a seasonal autoregressive component. The failure of the autocorrelation function to truncate at other lags may imply that a non-seasonal autoregressive term is required.

\subsubsection{Parameter Estimation}

This is the step in which estimations of parameters are required. There are three methods for estimating the parameters. These are the moment method, least-square estimation, and maximum likelihood estimation method. If the model is an AR (p) process, we can estimate by using the Yule-Walker equation. However, for both the AR (p) and MA (q) processes, it is possible to estimate the sample autocorrelation function. The estimation-stage results are used to check the parameter estimates and the appropriateness of the coefficient estimates, which includes the statistical significance of the estimated coefficient and standard error.

In time series analysis, there may be several adequate models that can be used to represent a given data set, and hence, numerous criteria for model comparison have been introduced in the literature. One of them is based on the so-called information criteria. The idea is to balance the risks of underfitting (selecting an order smaller than the true order) and overfitting (selecting an order larger than the true order).

Akaike (1978) introduced a criterion called the Akaike information criterion (AIC) in the literature. The AIC is a mathematical selection criterion for model building. When there are several competing models to choose from, select the model that gives the minimum of the AIC defined by (Shumay and Stoffar, 2010):

$$
A I C=\log \left(\delta_{k}^{2}\right)+\frac{n+2 k}{n}
$$


Where, $\boldsymbol{\delta}_{\boldsymbol{k}}^{2}=\frac{\boldsymbol{S} \boldsymbol{S} \boldsymbol{E}_{K}}{n}$ denotes the maximum likelihood estimator for the error variance and $\mathrm{k}$ is the number of seasonal and non-seasonal autoregressive and moving average parameters to be estimated in the model, that is, according to Wei (1990), $\mathrm{k}=\mathrm{p}+\mathrm{q}+\mathrm{P}+\mathrm{Q}+1$ and $n$ is the number of observations. The optimal order of the model is chosen by the value of $k$, which is a function of $p, q, \mathrm{P}$, and $\mathrm{Q}$ so that the value of $\mathrm{k}$ yielding the minimum AIC specifies the best model. Wei (1990) expressed the need to select a model that has fulfilled all the diagnostic checks and has as few parameters as possible in terms of parsimony.

\subsubsection{Diagnostic checking}

After fitting a provisional time series model, we can assess its adequacy in various ways. We check residuals of the estimate of the ARIMA model(s) to see if they are white noise; the autocorrelation function $(\mathrm{ACF})$ pick the best model with well-behaved residuals. Once we have identified a tentative model, the next step is to determine the adequacy of the model. Several diagnostic statistics and plots of the residuals can be used to examine the goodness of fit of the tentative model to the data.

The first approaches that can be used to evaluate the adequacy of a model are the plot of the errors over time, which can be written (Shumay and Stoffar 2010)

$$
\varepsilon_{t}=\frac{\left(y_{t}-y_{t}^{t-1}\right)}{\sqrt{p_{t}^{t-1}}}
$$

Where $\left(y_{t}-y_{t}^{t-1}\right)$ the one-step-ahead prediction of $y_{t}$ is based on the fitted model, and $\sqrt{p_{t}^{t-1}}$ is the estimated one-step-ahead error variance.

If visual inspections of the errors reveal that they are randomly distributed over time, then we have a good model.

The autocorrelations function (ACF) of the series can also be used to examine whether the residual of the fitted model is white noise or not. If the ACF is significantly different from zero, this implies that there is a dependence between observations (Janacek and Swift, 1993; Ferguson et al., 2000). There are different applications related to the Residual ACF (RACF) for the independence of residuals. The first one is the correlogram drawn by plotting $r_{k}(\varepsilon)$ against lag k.

$$
r_{a k}=\frac{\sum_{t=k+1}^{n} \varepsilon_{t} \varepsilon_{t-k}}{\sum_{t=1}^{n} \varepsilon_{t}^{2}}
$$


Under the assumption that the residual follows a white noise process, the standard errors of these $\left(r_{a k}\right)$ are approximately equal to $1 / \mathrm{T}$. Thus, under the null hypothesis that residual follows a white noise process, roughly $95 \%$ of the autocorrelation coefficient $\left(r_{a k}\right)$ should fall within the range $\pm 1.96 / \mathrm{T}$. If more than $5 \%$ of the coefficient falls outside this range, then the most likely residual does not follow a white noise process (Lehmann and Rode, 2001).

Ljung-Box Q (LBQ) Statistic: The Ljung-Box Q statistic can be employed to check the independence of the residual instead of visual inspection of the sample autocorrelations. A test of the hypothesis can be done for the model adequacy by choosing a level of significance and then comparing the value of calculated $x^{2}$ with $x^{2}$ critical value. A useful test in these concepts is the portmanteau lack of fit test.

This uses the entire residual sample with the null hypothesis that Ho: $\rho_{1}=\rho_{2}=\ldots=\rho_{k}$ The test statistic is calculated using the following equation (Ljung and Box, 1978):

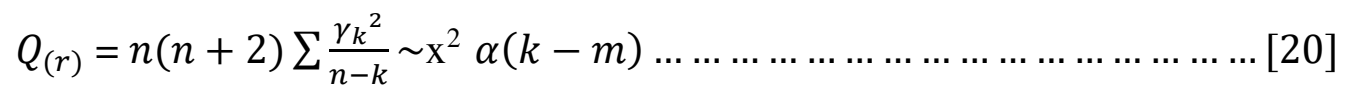

where $\mathrm{n}$ is the number of observations in the original time series, $\gamma_{k}^{2}$ is is the sample autocorrelation of the residuals at lag $k$, and $k$ is the number of lags.

Under the null hypothesis of model adequacy, Ljung and Box (1978) show that the Q - statistic approximately follows the $x^{2}(\mathrm{k}-\mathrm{m})$ distribution, where $\mathrm{m}$ is the number of parameters estimated. If a model is correctly specified, residuals should be uncorrelated and $Q_{(r)}$ should be small (pvalue should be large).

Test for normality of the residuals: If a data set is distributed according to the bell-shaped curve of the normal distribution, this set can be referred to as normal. Therefore, the histogram and Q-Q plot of a data set provides information related to normality. The null hypothesis for any test of normality is that the data are normally distributed. In this study, the Shapiro-Wilk test will be used for the diagnostic checking of residuals for normality. The Shapiro-Wilk statistic "W" is proportional to the ratio of the squared slope of the normal probability plot to the usual mean square estimate (Gibbons, 1994):

$$
W=\frac{\left(\sum_{i=1}^{n} a_{i, n} * x_{i}\right)^{2}}{\sum_{i=1}^{n}\left(x_{i}-\bar{x}\right)^{2}} .
$$


Where: $a_{i, n}$ for $\mathrm{W}$-statistic is given in the table.

\subsubsection{Forecasting}

The last step of the ARIMA modeling process is forecasting. In forecasting, the goal is to predict future values of a time series variable, $\mathrm{x}_{\mathrm{t}+\mathrm{k}}, \mathrm{k}=1,2 \ldots$ based on the data collected to the present, $\mathrm{y}=\left\{\mathrm{y}_{\mathrm{t}}, \mathrm{y}_{\mathrm{t}-1} \ldots \mathrm{y}_{1}\right\}$. Throughout this section, we assume that $\mathrm{y}_{\mathrm{t}}$ is stationary and that the model parameters are known.

\subsubsection{Measures forecasting Accuracy}

Once forecasts are made, they can be evaluated if the actual values of the series to be forecasted are observed. There are some measurements of the accuracy of forecasts. These are the root mean square error (RMSE), mean absolute error (MAE), mean absolute percentage error (MAPE), and Theil's inequality coefficient (Theil-U).

If $Y_{t}$ is the actual observation for period $\mathrm{t}$ and $F_{t}$ is the forecast for the sample period, then the error is defined as

$$
U_{t}=Y_{t}-F_{t}
$$

Usually, $F_{t}$ is calculated using data $Y_{1} \ldots Y_{t-1}$. It is a one-step forecast because it is forecasting one period ahead of the last observation used in the calculation. Therefore, we describe $U_{t}$ a onestep forecast error. It is the difference between the observation $Y_{t}$ and forecast made using all observations up to but not including $Y_{t}$.

If there are observations and forecasts for $\mathrm{T}$ periods, then there will be $\mathrm{n}$ error terms, and the following standard statistical measures can be defined:

$$
\begin{aligned}
& \text { Mean Error }(\mathrm{ME})=\frac{\sum_{t=1}^{T} U_{t}}{T} \ldots \ldots \ldots \ldots \ldots \ldots \\
& \text { Mean Absolute Error }(\mathrm{MAE})==\frac{\sum_{t=1}^{T}\left|U_{t}\right|}{T} \ldots \\
& \text { Mean Squared Error }(\mathrm{MSE})=\frac{\sum_{t=1}^{T} U_{t}{ }^{2}}{T} \ldots \ldots
\end{aligned}
$$

Equation [22] can be used to compute the error for each period. These can then be averaged as in equation [23] to give the mean error. However, the ME is likely to be small because positive and negative errors tend to offset one another. The ME will only tell you if there is systematic under- 
or-over forecasting, called the forecasting bias. This does not give much indication of the size of the typical errors.

Therefore, the MAE is defined by first making an error positive by taking its absolute value, and then averaging the results. The idea behind the definition of MSE is similar. Here, the errors are made positive by squaring each one, and then the squared errors are averaged. MSE has the advantage of being more interpretable and is easier to explain than non-specialists.

Each of these statistics deals with measures of accuracy whose size depends on the scale of the data. Therefore, they do not facilitate comparison across different time series and for different time intervals. To make comparisons, we need to work with relative or percentage error measures. First, let us define a relative or percentage error as

$$
\mathrm{PE}_{\mathrm{t}}=\left(\frac{Y_{t}-F_{t}}{Y t}\right) \times 100
$$

Then the following two relative measures are frequently used:

$$
\begin{aligned}
& \text { Mean Percentage Error }(\mathrm{MPE})=\frac{\sum_{t=1}^{T} P E_{t}}{T} \ldots \ldots \ldots \ldots \ldots \ldots \ldots \ldots \ldots \ldots \ldots \ldots \ldots \ldots \ldots \ldots \ldots \ldots \ldots \ldots \ldots \ldots \ldots \ldots \ldots \\
& \text { Mean Percentage Absolute Error }(\mathrm{MPAE})=\frac{\sum_{t=1}^{T}\left|P E_{t}\right|}{T} \ldots \ldots \ldots \ldots \ldots \ldots \ldots
\end{aligned}
$$

Equation [26] can be used to compute the percentage error for any period. These can be averaged as in equation [27] to give the mean percentage error. However, as with the ME, the MPE is likely to be small because positive and negative PEs tend to offset one another. Hence, MAPE is defined using the absolute values of PE in equation [28].

Alternatively, Theil's U statistic can be used as a measure of forecasting accuracy. LikeMAPE statistics, high values suggest poor performance in the forecast. However, and unlike MAPE, the U-Theil corrects the performance scale that MPAE had. Theil's U can be estimated as

$$
U=\frac{\sqrt{\frac{\sum_{t=1}^{n}\left(Y_{t-F_{t}}\right)^{2}}{n}}}{\sqrt{\frac{\sum_{t=1}^{n} F_{t}^{2}}{n}}+\sqrt{\frac{\sum_{t=1}^{n} Y_{t}^{2}}{n}}} \ldots \ldots \ldots \ldots \ldots \ldots \ldots \ldots \ldots \ldots \ldots \ldots \ldots
$$

The scaling of $U$ is such that it will always lie between 0 and 1 . If $U=0, Y_{t}=F_{t}$ for all forecasts and there is a perfect fit; if $U=1$, the predictive performance is not good. 


\section{RESULTS AND DISCUSSIONS}

The study is based on monthly time series data observed from January 2012 to December 2016. The total number of observations was 60 . The discussion begins by describing the data set and the results of the model selection procedure. Then, the results are interpreted and discussed. Finally, the mean monthly temperature in the Assosa district is forecasted. The statistical software package used for the analysis was R-3.6.3.

\subsection{Descriptive Statistics}

Some descriptive statistics, including the mean, median, standard deviation, minimum, and maximum values of the mean monthly temperature series are presented in Table 3.1.

Table 3.1Descriptive statistics

\begin{tabular}{|l|l|l|l|l|l|l|}
\hline Variable & $\mathrm{N}$ & Mean & Median & StDev & Min & Max \\
\hline Mean monthly Temperature $\left({ }^{\circ} \mathrm{C}\right)$ & 60 & 28.025 & 27.6 & 2.996 & 23.3 & 33.2 \\
\hline
\end{tabular}

Table3.1 Descriptive statistics for Assosa maximum monthly temperature data. From the above table, the minimum and maximum temperature Assosa was $23.3^{\circ} \mathrm{Cand} 33.2^{\circ} \mathrm{Crespectively.} \mathrm{The}$ average temperature of the town for ten years was $28.025^{\circ} \mathrm{C}$.

\subsection{Testing Stationarity}

Visual Inspection: Regardless of which technique is used, the first step in any time series analysis is to plot the observed values against time. Several qualitative aspects are noticeable, as you visually inspect the graph. Figure 3.1 below shows the mean monthly temperatures recorded over the years from January 2012 to December 2016. The pattern of the time series plot in Figure-3.1 below does not show any systematic upward and downward change about the mean, that is, it shows no particular trend. This indicates that the series is non-seasonally stationary. However, by critical observations year by year, it is clear that there existed a sharp rise and fall in the mean temperatures recorded. Besides, from the autocorrelation function plot in Figure-3.2 below, the presence of seasonality(periodic effect) behavior of the series is clear. Therefore, from 
the time series plot and autocorrelation plot (Figure-3.1 and Figure-3.2) below, it can be observed that our series has seasonal variation, and seasonal differencing is needed to make it stationary.

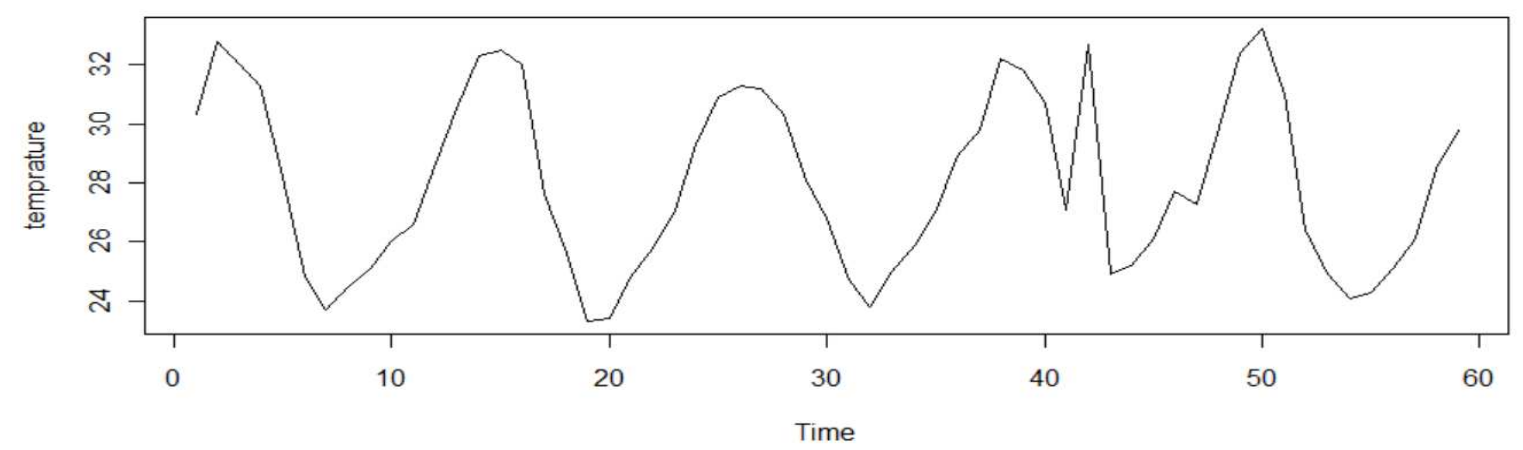

Figure-3.1 Time series plot

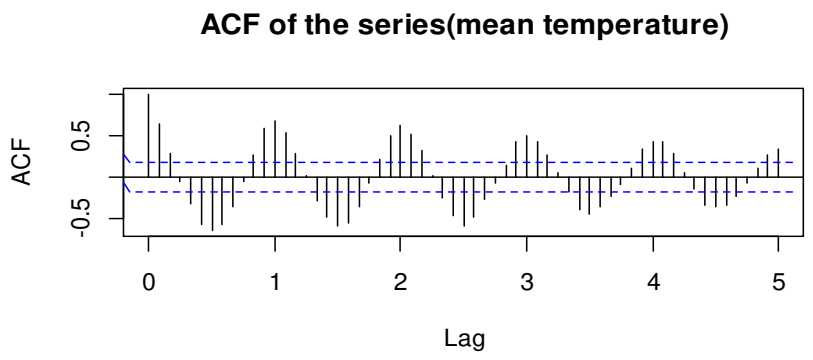

Figure-3.2 Autocorrelation plot

Unit root test: The test first imposes that the series has a unit root, versus the series is stationary. We test the null hypothesis using the Augmented Dickey-Fuller (ADF) test. This test is used to know whether our series is stationary or not.

Table 3.2: Summary of ADF unit-roots test for original series and the second difference of the series

\begin{tabular}{|l|l|l|l|l|l|}
\hline Series & $\begin{array}{l}\text { ADF-test } \\
\text { statistic }\end{array}$ & $\begin{array}{l}1 \% \\
\text { critical } \\
\text { value }\end{array}$ & $\begin{array}{l}5 \% \\
\text { critical } \\
\text { value }\end{array}$ & $\begin{array}{l}10 \% \\
\text { critical } \\
\text { value }\end{array}$ & Decision \\
\hline $\begin{array}{l}\text { Original } \\
\text { Series }\end{array}$ & -8.46 & -3.51 & -2.96 & -2.60 & $\begin{array}{l}\text { Don't reject } \\
\text { the null }\end{array}$ \\
\hline
\end{tabular}




\begin{tabular}{|l|l|l|l|l|l|}
\hline Seasonallydifferenced & -2.51 & -3.51 & -2.93 & -2.60 & $\begin{array}{l}\text { Rypothesis } \\
\text { null the } \\
\text { hypothesis }\end{array}$ \\
\hline
\end{tabular}

According to the results in the table, the null hypothesis, which states the data needs to be differenced for the original series should not be rejected as the test statistics are less than the critical value at a specified significance level. However, as also suggested by the visual analysis of the ACF plot (Figure-3.3) of the series, after seasonal differencing, the seasonal stationarity property of the series is satisfied as the test statistic is greater than the given critical values.

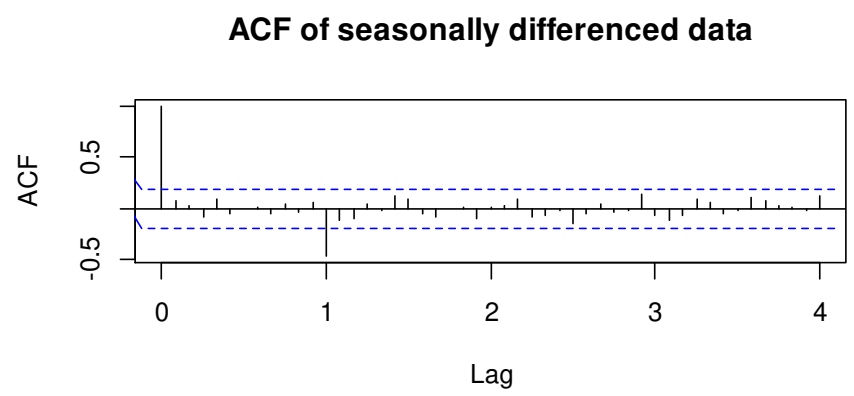

Figure-3.3ACF plot

\subsection{Model identification}

Once the degree of difference has been determined, the autoregressive and moving average orders are selected by examining the sample autocorrelation and sample partial autocorrelation. To use the sample autocorrelation and sample partial autocorrelation functions for tentative model parameter identification, we consider the ACF and PACF. Using Table-3.1 and Table-3.2 as a guide, the preliminary values of $\mathrm{p}, \mathrm{q}, \mathrm{P}$, and $Q$ are chosen. Because we are dealing with estimates, it will not always be clear whether the sample ACF or PACF is tailing off or cutting off. Besides, two models that are seemingly different can be very similar (Shumway and Stoffer, 2010). With this in mind, we should not worry about being so precise at this stage of the model fitting. At this stage, a few preliminary values of p,q, P, and Q should be at hand, and we can start estimating the parameters. 


\section{ACF of seasonally difference series}

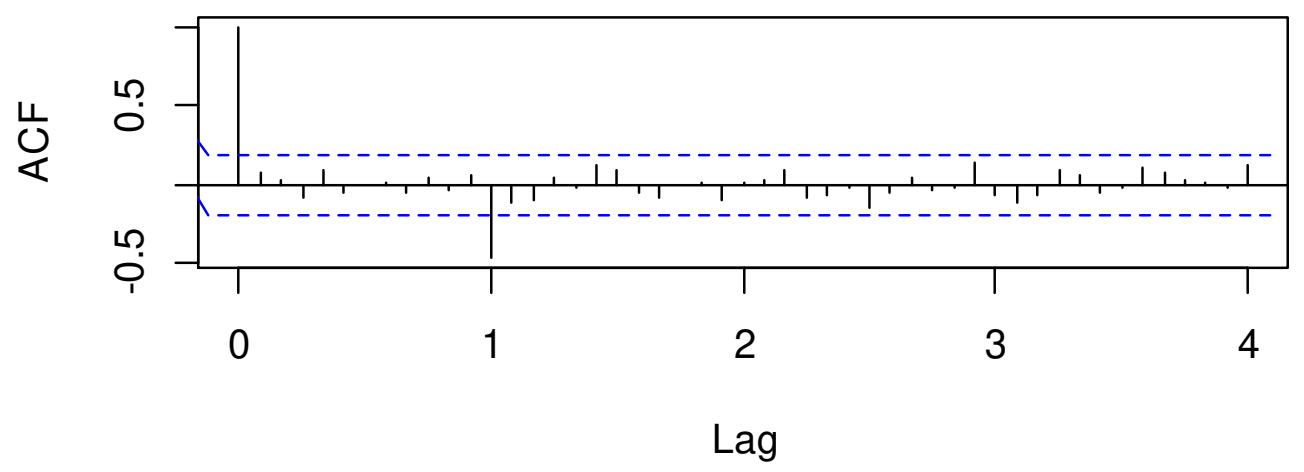

PACF of seasonally difference series

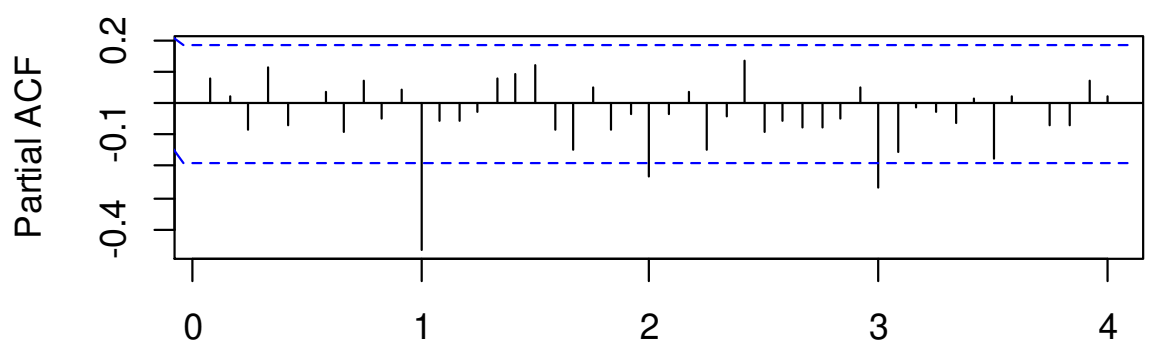

Lag

Figure-3.4 Seasonally Difference series

From Figure-3.4 above, the ACF at lag-1 is significantly different from zero since the spike passed out of the confidence limit. Hence, the order of the non-seasonal MA term is 1 and that of the seasonal MA term is occurring at lag which is multiple of 12 . There is no significant spike after lag-1 in ACF. Hence, the possible order of the seasonal MA term is 0. Similarly, significant spikes in the PACF (at low lags), i.e., lags=1, 2, and 3, indicated possible non-seasonal AR terms. Hence, the order of non-seasonal terms is 3 and that of seasonal terms is 0 as there is no significant spike after lag-3 in PACF.

By fitting the four possible models, we select the best model by using the AIC.

Table 3.4 AIC Model selection for temperature data

\begin{tabular}{|l|l|l|}
\hline ARIMA & AIC Values & Log likelihood \\
\hline ARIMA $(0,0,1)$ & 271.55 & -132.78 \\
\hline
\end{tabular}




\begin{tabular}{|l|l|l|}
\hline $\operatorname{ARIMA}(3,0,0)$ & 238.02 & -114.01 \\
\hline $\operatorname{ARIMA}(3,0,1)$ & 224.02 & -106.01 \\
\hline $\operatorname{ARIMA}(2,0,0)$ & 252.98 & -122.49 \\
\hline ARIMA $(2,0,1)$ & 230.62 & -110.31 \\
\hline ARIMA(1,0,0) & 256.53 & -125.26 \\
\hline ARIMA(1,0,1) & 256.15 & -124.07 \\
\hline
\end{tabular}

From Akaike criteria's points of view, the proper model to best fit the data isSARIMA $(3,0,1)$ as it has the smallest value of AIC which is 224.02, and maximum value of log-likelihood which is -106.01 .

\subsection{Parameter estimation for temperature data}

Once the order of ARIMA (p, d, q), the model has been specified, and the next step is the estimation of parameters.

\section{Table 3.5 Estimates of Parameters}

\begin{tabular}{|c|l|l|}
\hline parameter & estimate & Std.Error \\
\hline$\alpha_{1}$ & 2.017 & 0.1207 \\
\hline
\end{tabular}

\subsection{Diagnostic checking}

In this section, how well the selected model fits the actual mean monthly temperature data will be assessed. If the model fits the data well, the residuals of the fitted model are random (Chatfield, 1991). In ARIMA modeling, the selection of the best model to data is directly related to how well the residual analysis is performed (Kadri et al., 2005). Therefore, several diagnostic statistics and plots of the residuals can be used to examine the goodness of fit of the selected model to the data.

The first step in this stage is to plot the standardized residuals of series. If the model fits well, the standardized residuals should behave as an identically and independently distributed sequence with mean zero and variance one (Shumay and Stoffar, 2010). The time plot should be inspected 
for any clear departure from this assumption. The inspection of the time plot of the standardized residuals for the fitted model in Figure-4.4 below shows no patterns.

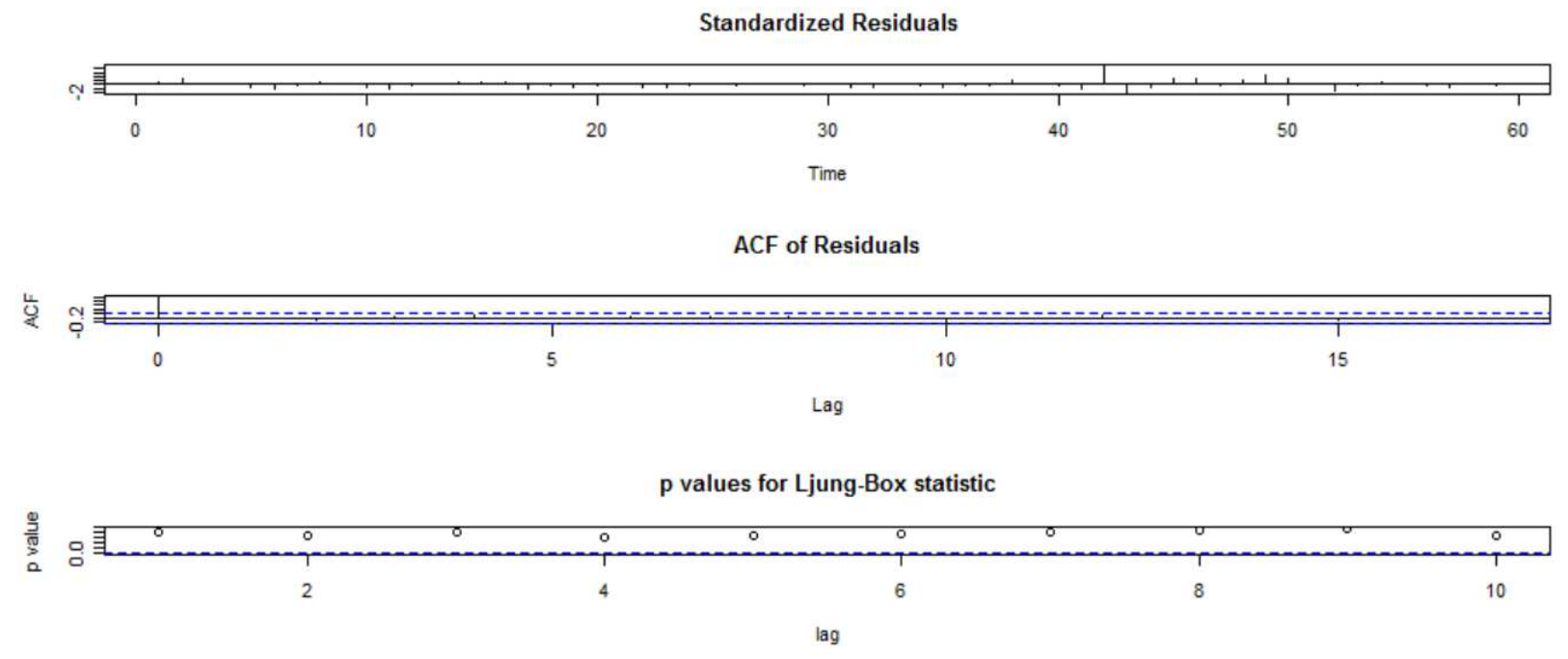

Figure 3.4 Diagnostics of the residuals from the fitted model

The Ljung-Box Q-statistic: This is the formal test used to check whether the series is independent or not; here, we can perform a general test that takes into consideration the magnitudes of residual ACF as a group instead of individual visual inspection of the sample autocorrelations. A test of this hypothesis can be done for the model adequacy by choosing a level of significance and then comparing the value of the calculated chi-square with the critical value. The Q-statistic for each group of six lags was computed using Eq. (19) and the results are summarized in Table 3.6 and figure-3.5 below.

Table 3.5: Residual white noises check with the Ljung-Box test for the fitted model

\begin{tabular}{|l|l|l|l|l|}
\hline Lag & Q-statistics & DF & $\begin{array}{l}\text { Critical value at 5\% level of } \\
\text { significance }\end{array}$ & P-value \\
\hline 6 & 3.1934 & 5 & 12.592 & 0.6702 \\
\hline 12 & 7.1977 & 11 & 19.675 & 0.7829 \\
\hline 18 & 12.328 & 17 & 27.587 & 0.7799 \\
\hline 20 & 15.292 & 19 & 30.144 & 0.7039 \\
\hline
\end{tabular}


From Table-3.5, it can be observed that the Q-statistics are less than the critical value, and the pvalue is greater than 0.05 at specified lags, which implies that the white noise hypothesis is not rejected.

Table-3.6 the result of the Normality test for residual of the fitted model using the Shapiro-wilk test.

\begin{tabular}{|l|l|l|}
\hline Shapiro-Wilk & Test statistic & p-value \\
\cline { 2 - 3 } Test statistic & 0.90796 & 0.5152 \\
\hline
\end{tabular}

From the above table, the p-value is greater than 0.05, indicating the residuals of the fitted models are normally distributed. In addition to these tests, Figure -4.5 shows the histograms and QQ-plot of the residuals. As expected, the curves significantly reflect a normal distribution.

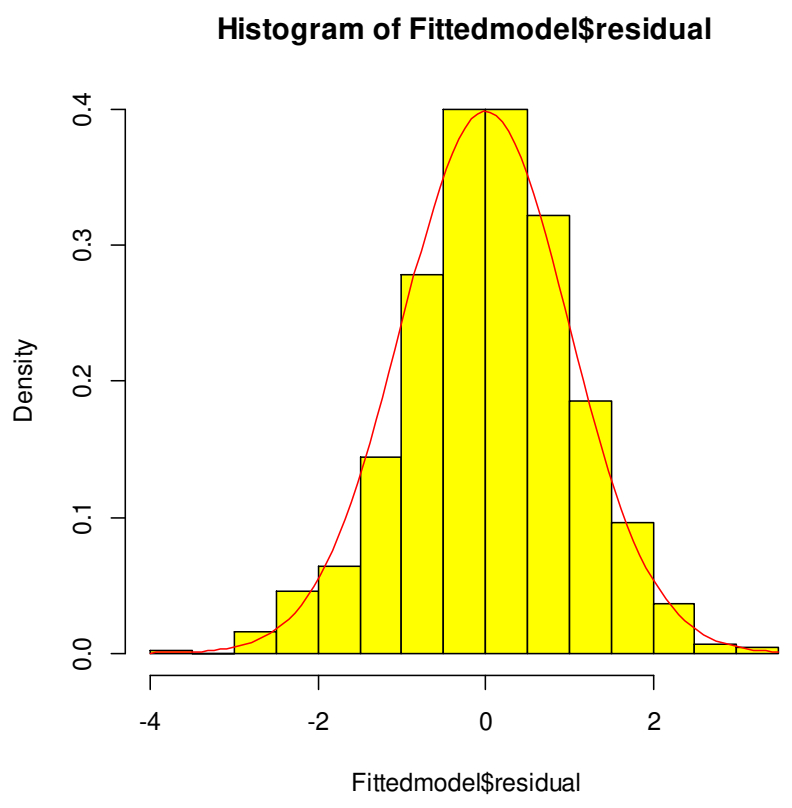




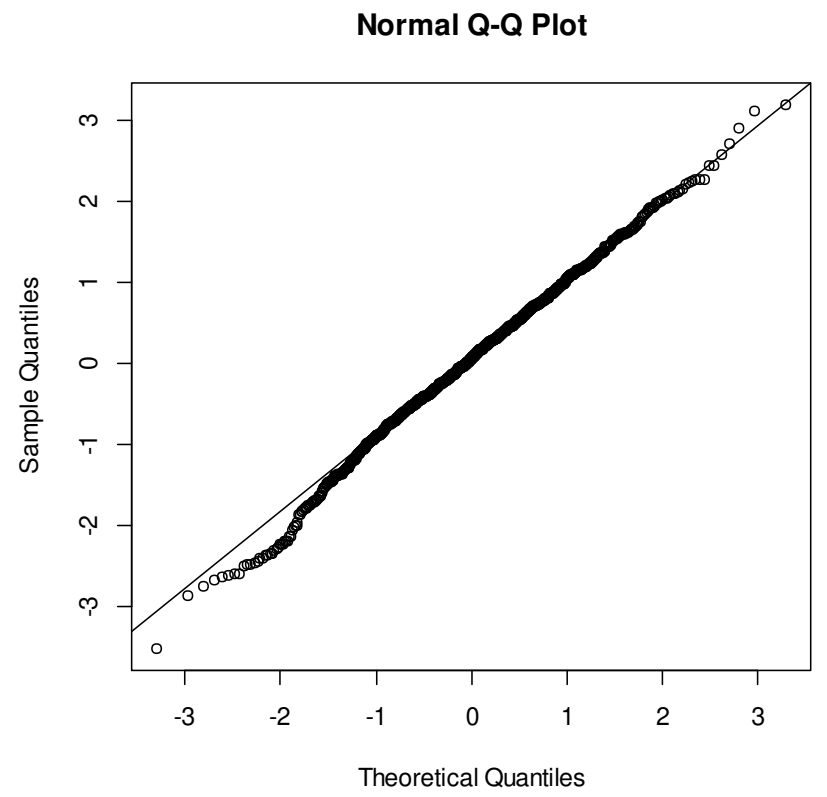

Figure 3.5 Diagnostic tests

\subsection{Forecasting}

Since the model diagnostic tests show that all the parameter estimates are significant and the residual series is white noise, the estimation and diagnostic checking stages of the modeling process are complete. We can now proceed to forecast the series with fitted.

Forecasting refers to the process of predicting or forecasting future mean monthly temperature values from a known time series. From equation [11], the ARIMA $(3,0,1)$ can be written as:

$$
\left(1-B^{12}\right) \alpha(B) y_{t}+\varepsilon_{t}=0
$$

Where, $\alpha(B)=1-\alpha_{1} \mathrm{~B}$, then we have:

$$
\left(1-B^{12}\right)\left(1-\alpha_{1} B\right) y_{t}+\varepsilon_{t}=0
$$

The above equation can be expanded to give;

$$
y_{t}=\alpha_{1}\left(y_{t-1}-y_{t-14}\right)+y_{t-13}-\varepsilon_{t}
$$


Where $\varepsilon_{t}$ is replaced by zero since it is white noise.

Lastly, by substituting

Lastly, by substituting $\alpha_{1}$ by its estimate, we obtain;

$$
\begin{gathered}
\hat{y}_{t}=\hat{\alpha}_{1}\left(y_{t-1}-y_{t-14}\right)+y_{t-13} \\
\hat{y}_{t}=2.017\left(y_{t-1}-y_{t-14}\right)+y_{t-13}
\end{gathered}
$$

Where, $y_{t-1}, y_{t-13}$ and $y_{t-14}$ are observations at lag -1, lag-13, and lag-14 respectively.

The above equation can be written in the form that is used for forecasting as follows:

$$
\hat{y}_{t+m}=2.017\left(y_{t-1+m}-y_{t-14+m}\right)+y_{t-13+m}
$$

Table 3.7 Forecasted mean temperature of 2017 using ARIMA $(3,0,1)$

\begin{tabular}{|l|l|l|l|l|l|l|l|l|l|l|l|l|}
\hline Year & Jan & Feb & Mar & Apr & May & Jun & Jul & Aug & Sep & Oct & Nov & Dec \\
\hline 2017 & 31.147 & 31.28 & 30.66 & 29.31 & 27.73 & 26.32 & 25.46 & 25.34 & 25.93 & 27.05 & 28.34 & 29.46 \\
\hline
\end{tabular}

\subsubsection{Evaluation of accuracy}

If the fitted ARIMA $(3,0,1)$ model has to perform well in forecasting, the forecast error will be relatively small. The accuracy of forecasts is usually measured using root mean square error (RMSE), mean absolute error (MAE), mean absolute percentage error (MAPE), and Theil's inequality coefficient (Theil-U). The result shows that the Mean Absolute Percentage Error (MAPE) is $5.20 \%$, which is relatively low and Theilee $\mathrm{s}$ inequality coefficient (U-statistic) is 0.056, which lies between zero and one.

Table 3.6 Summary of forecasting accuracy statistics

\begin{tabular}{|l|l|}
\hline \multicolumn{2}{|c|}{ Accuracy measures } \\
\hline Root Mean Square Error (RMSE) & 1.59 \\
\hline Mean Absolute Error(MAE) & 1.064 \\
\hline Mean Absolute Percentage Error(MAPE) & 5.20 \\
\hline
\end{tabular}




\section{CONCLUSIONS AND RECOMMENDATIONS}

In this study, ten years mean monthly temperature records were analyzed, using data from the metrological stations of Assosa, mainly to study the mean monthly temperature pattern in the Assosa district. Box-Jenkins methodology was used to fit the Seasonal ARIMA model to the data.

The pattern of mean monthly temperatures in the Assosa district from 2012 to 2016 was observed to be free of the trend pattern. The stationarity of the mean temperature series was verified by the time plot of the series as well as the use of the ADF test. However, regular mean temperature values were recorded every year, hence the presence of seasonally non-stationary of the series were observed through the time plot of the series and ACF of the series.

The seasonal component of the series was removed through the first seasonal differencing. Following the procedures of Box Jenkins's ARIMA model building, several suggested models were developed. However, based on the computed AIC values for each of the suggested models, the best model was derived as ARIMA $(3,0,1)$. However, model diagnostics were performed through the careful performance of the model residuals. The model residuals were found to be following a white noise process with a mean of zero and a constant variance, hence uncorrelated. Also, the model residuals were found to be near normality through the use of the Shapiro-Wilck test and plots of histograms and Q-Q plot. Based on the model diagnostics performed, the identified model was found to be adequate and good for predicting future mean temperatures in the Assosa district. The forecasted mean temperature values showed a similar pattern to previous recordings.

Since temperature change is caused mainly by human activities, urgent actions are needed to tackle the problem. To combat the change of temperature the following points are recommended.

- It is necessary to conserve forests and other terrestrial ecosystems.

- Development planners should design strategies and plans by taking into account an increasing temperature impact on rural livelihood. 
- The government needs to promote national, regional, and international collaborations and networking on the issues.

Generally, strategies that involve conservation or improvement of vegetation cover are appropriate to tackle the problem of temperature change.

\section{ABBREVIATIONS}

$\mathrm{ACF}=$ Autocorrelation function

$\mathrm{ADF}=$ Augmented Dickey Fuller

$\mathrm{AIC}=$ Akaike Information Criterion.

$\mathrm{AR}=$ Auto Regressive

ARIMA = Autoregressive Integrated Moving Average.

ARMA = Autoregressive Moving Average

IPCC $=$ Intergovernmental Panel on Climatic Change

MA = Moving Average .

PACC $=$ Partial Autocorrelation Coefficients.

PACF $=$ Partial Autocorrelation Function

$\mathrm{PP}=$ Phillips-Perron

NMA = National Metrological Agency

\section{Declarations}

\section{Ethics approval and consent to participate}

Not applicable

\section{Consent for publication}

Not applicable

\section{Availability of data and materials}

Not applicable. All the data supporting the findings are contained within the manuscript. 


\section{Competing interests}

Authors declared that there was no competing interest.

\section{Funding}

No fund is found for this study.

\section{Authors' contributions}

THK conducted a literature search, planned the study, carried out data collection, performed data analysis and interpretation and drafted the manuscript. SGG reviewed the literature search, design of the study, data analysis and data interpretation. Both authors read and approved the final manuscript.

\section{Acknowledgements}

The authors would like to acknowledgement the metrological stations of Assosa district staff members.

\section{Authors' Information}

\section{Affiliations}

Department of Statistics, Samara University, Samara, Ethiopia Tigabu Hailu Kassa

Department of Statistics, Debre Tabor University, Debre Tabor, Ethiopia Shewayiref Geremew Gebremichael

\section{Corresponding author}

\section{Correspondence to Tigabu Hailu Kassa}




\section{REFERENCES}

Akaike, H., (1978). A Bayesian analysis of the minimum AIC procedure.

Gallop, C., Tseand, C., Zhao, J., 2012, A Seasonal Autoregressive Model of Vancouver Bicycle Traffic Using Weather Variables.TRB 2012 Annual Meeting.

Saez, M., Sunyer, J., Castellsague, J., Murillo, C., and Manto, J., 1994, Relationship between Weather Temperature and Mortality: A time series analysis approach in Barcelona. International Journal of Epidemiology, 24 (3), Pages 576-582.

Janacek, G. and Swift, L., (1993.) Time Series Forecasting, Simulation, Application, Ellis Horwood, New York, USA.

Tektax, M., 2010, Weather Forecasting Using ANFIS and ARIMA Models: A Case Study for Istanbul.

Granger and Newbold., (1986). Forecasting Economic Time Series; Academic Press, USA.

Chatfield, C. (2003).The Analysis of Time-series: An Introduction.

D. Ruppert, (2011) Statistics and Data Analysis for Financial Engineering, Springer Texts in Statistics,

Granville Tunnicliffe Wilson, 2016. "Time-Series Analysis: Forecasting and Control, 5th Edition, by George E. P. Box ,Gwilym M. Jenkins, Gregory C. Reinsel, and Greta M. Ljung, 2015 . Published by John Wiley, and Sons Inc., Hoboken, N," Journal of Time Series Analysis, Wiley Blackwell, vol. 37(5), pages 709-711, September.

Gibbons, R.D., (1994). Statistical Methods for Groundwater Monitoring. John Wiley \& Sons, New York.

Hamilton, J. D. (1994). Time series analysis. Princeton, N.J: Princeton University Press. 
Kendall, M. (1976) Time Series. 2nd Edition, Charles Griffin and Co Ltd., London and High Wycombe.

Wei, W.W.S., (1990).Time Series Analysis. Addison-Wesley Publishing Company, Inc., 478 pp., New York-USA.

Ljung, G.M. and Box, G.E.P., (1978).”On a Measure of Lack of Fit in Time Series Models", Biometrika, Vol.65,297-303pp.

Box, G. E. P., and Jenkins, G. M. (1976). Time series analysis: Forecasting and control. San Francisco: Holden-Day

Box, G.E.P., and Pierce, D.A., (1970). Distributions of residual autocorrelations in autoregressive integrated moving average models. J. American Stat. Assoc., Vol.72, 397-402pp.

Hipel, K.W., McLeod, A.I., and Lennox, W.C., (1977). Advances in Box-Jenkins Modeling, Model Construction", Journal of Water Resources Research, Vol.13, 567-575pp.

Shumway, R.H., and Stoffer, D.S.,(2010).Time series analysis and its applications with R Examples, Springer, $3^{\text {nd }}$ ed.

\section{Acknowledgements}

The authors would like to acknowledge the Assosa district metrological station staffs.

\section{Funding}

Not Applicable 


\section{Author information}

\section{Contributions}

Both authors have made a vital intellectual contribution to realize this study. THK has designed the study, collected and analyzed the data, interpreted the results, and wrote the draft manuscript. SGG has participated in designing the study, involved in data collection, analysis, and interpretation, structuring the manuscript; and provided critical comments and suggestions on the draft manuscript. Both authors read and approved the final manuscript.

\section{Corresponding author}

\section{Correspondence to Tigabu Hailu Kassa}

\section{Ethics declarations}

\section{Ethics approval and consent to participate}

Not applicable.

\section{Consent for publication}

Not applicable.

\section{Competing interests}

The authors declare that they have no competing interests. 
Figures

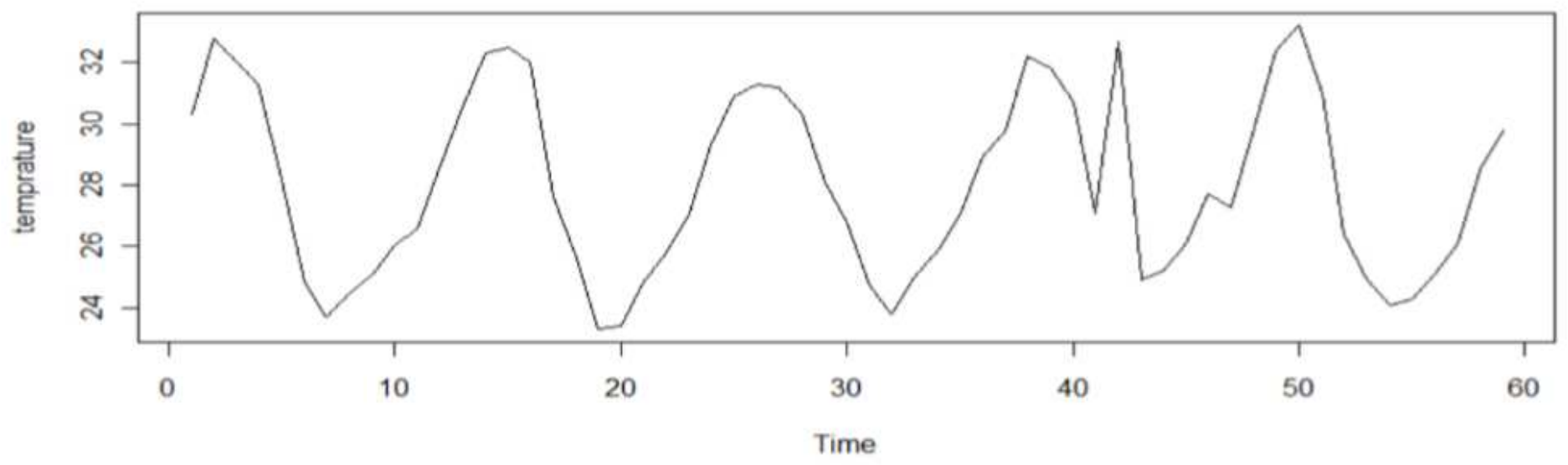

Figure 1

Time series plot

ACF of the series(mean temperature)

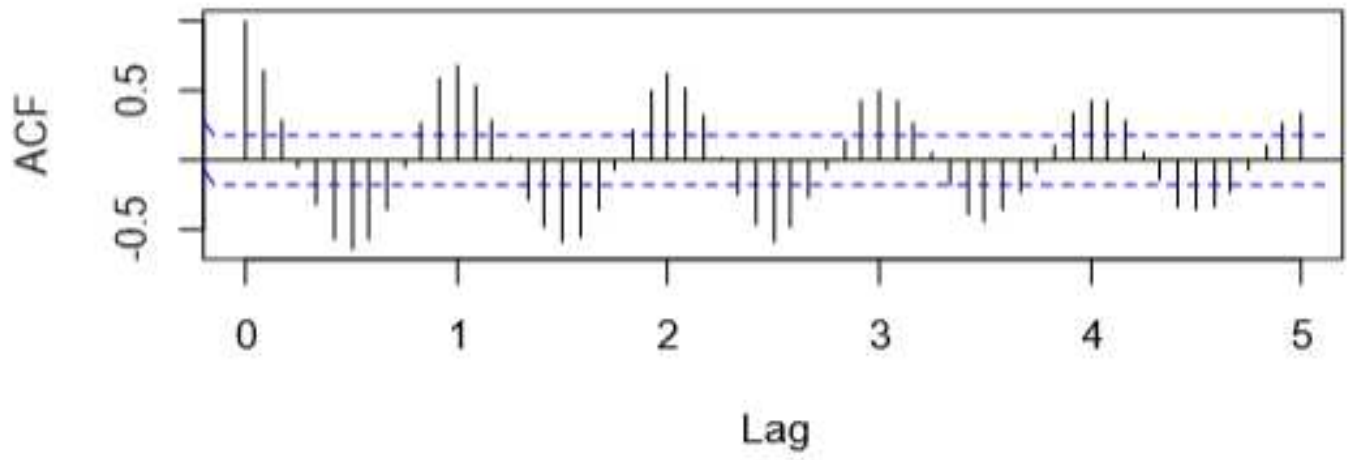

Figure 2

Autocorrelation plot 


\section{ACF of seasonally differenced data}

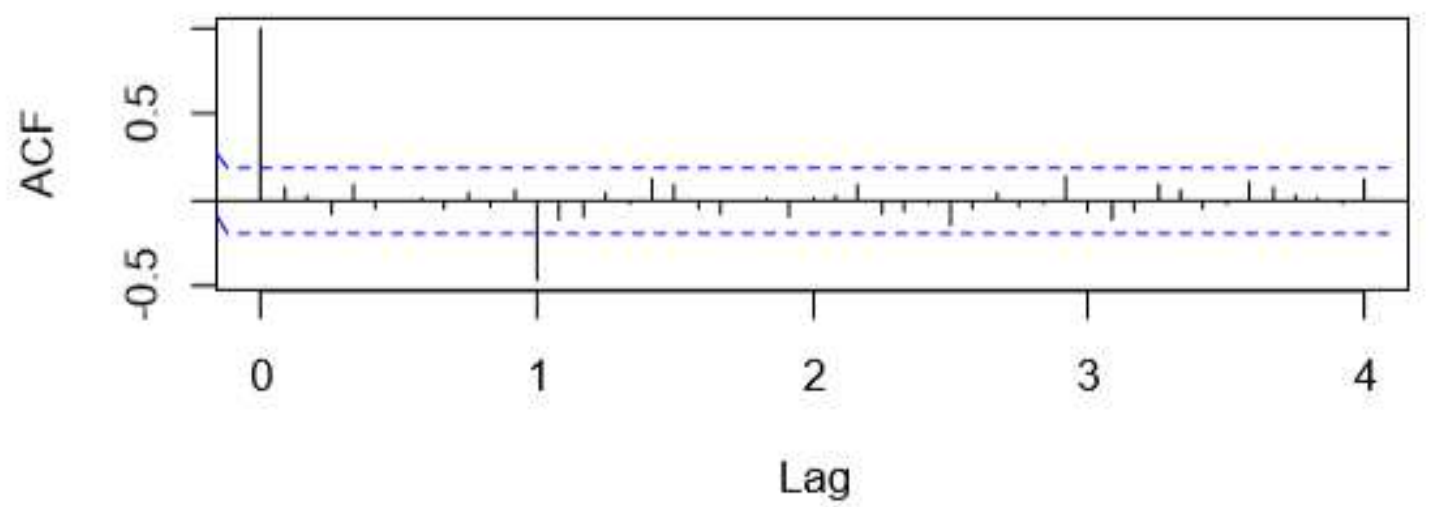

Figure 3

ACF plot 


\section{ACF of seasonally difference series}

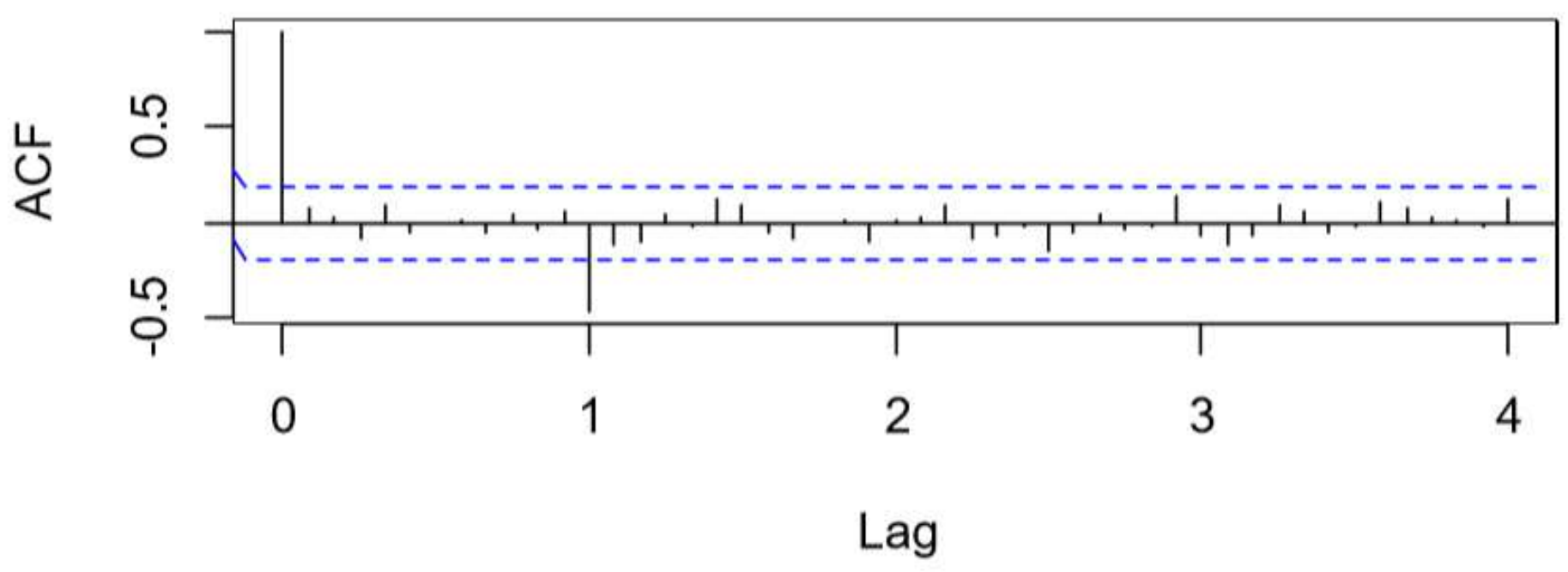

PACF of seasonally difference series

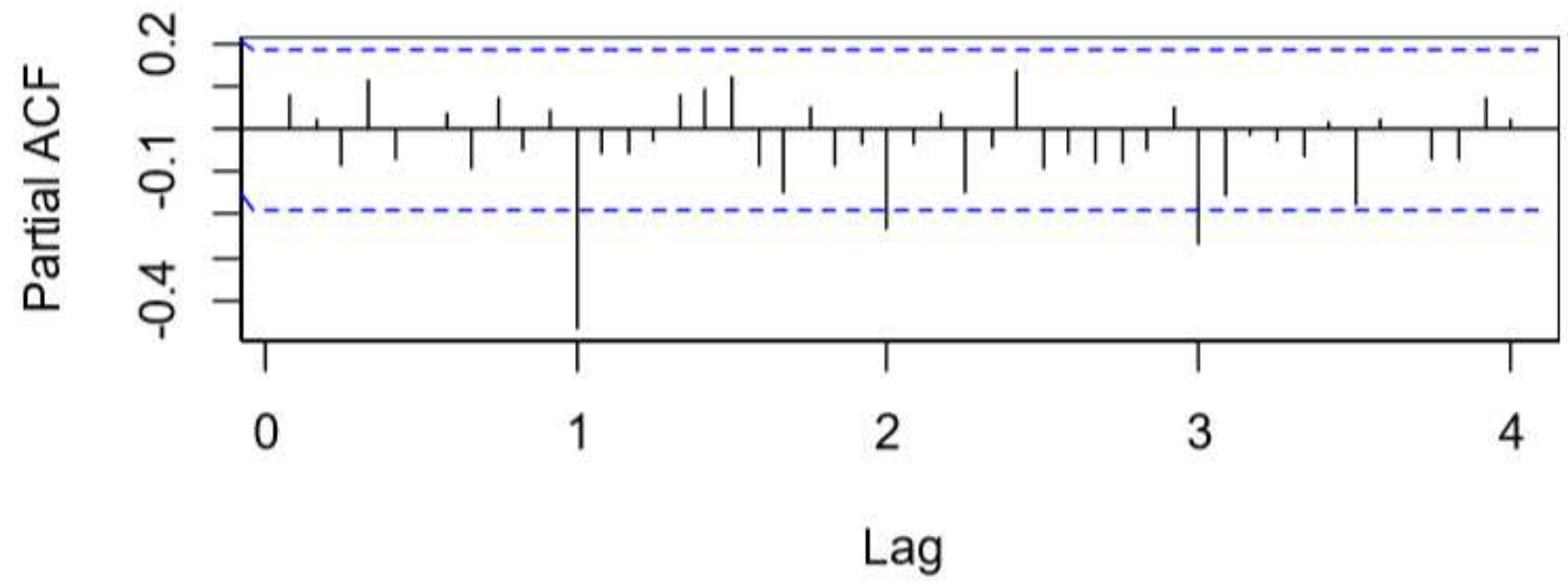

Figure 4

Seasonally Difference series 
Standardized Residuals

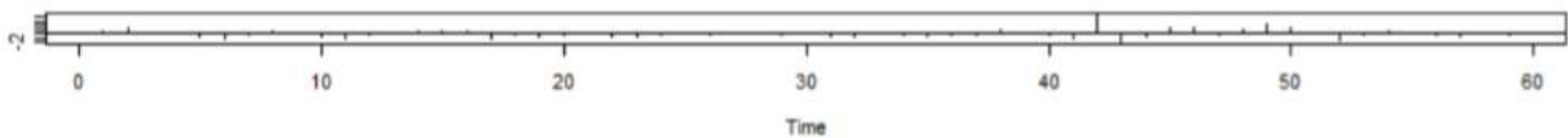

ACF of Residuals

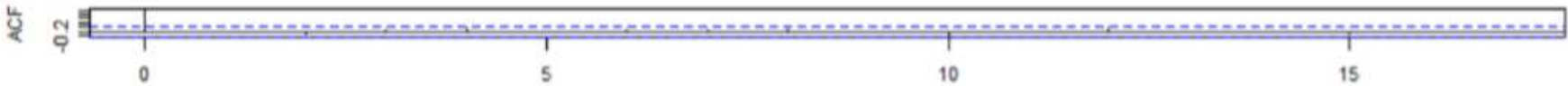

Lag

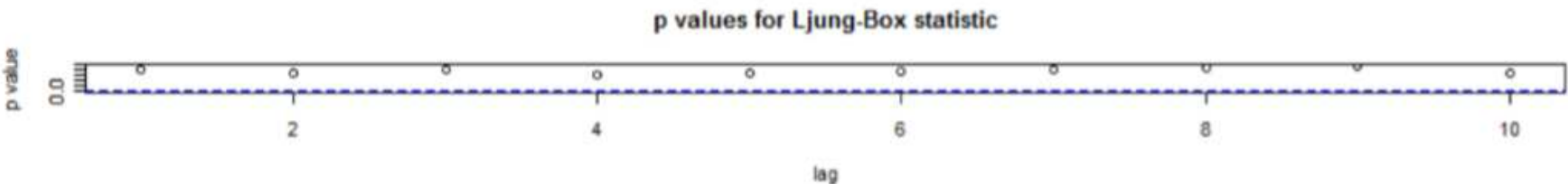

lag

Figure 5

Diagnostics of the residuals from the fitted model

Histogram of Fittedmodel\$residual

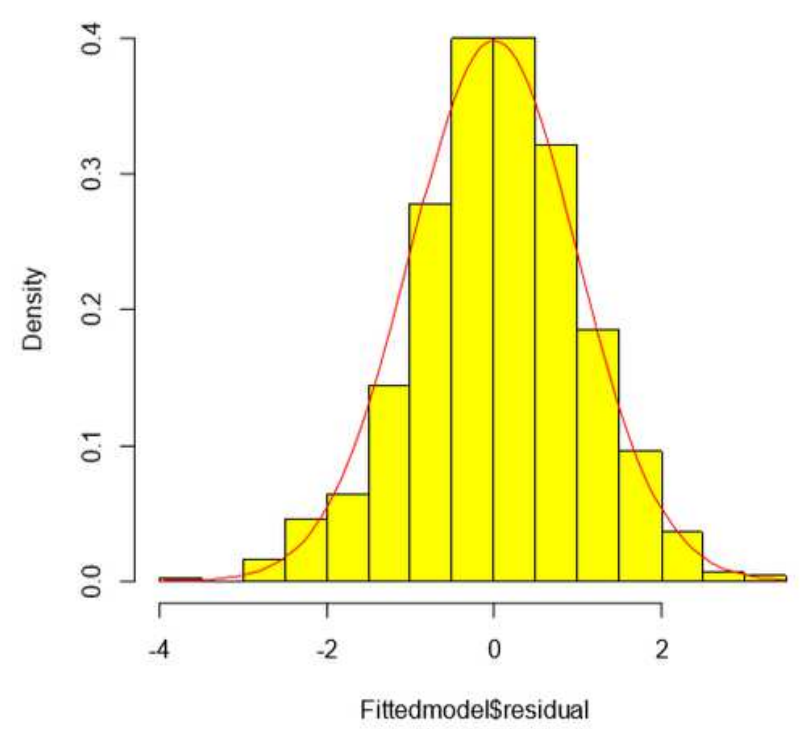

Normal Q-Q Plot

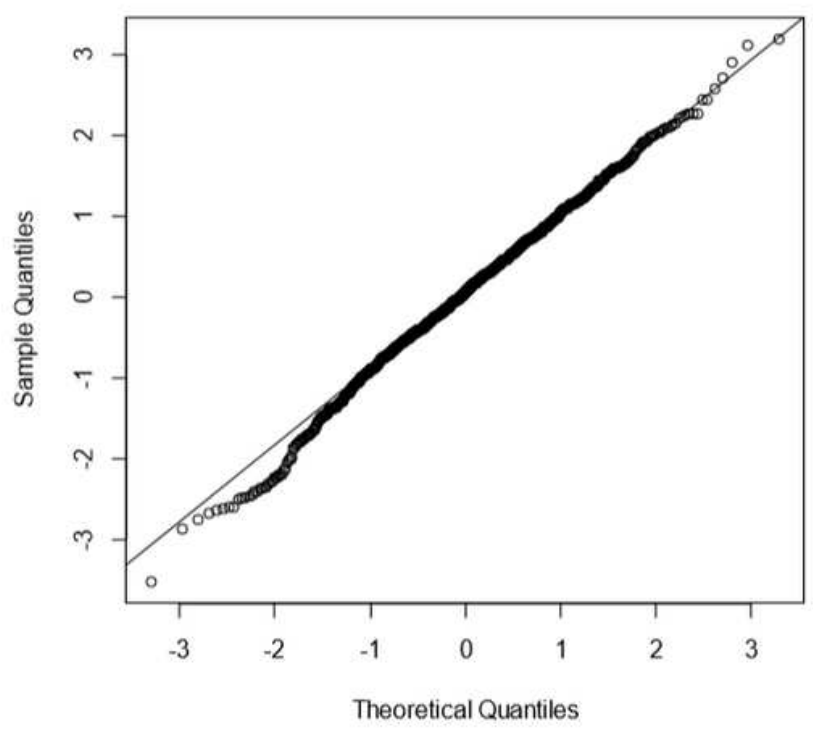

Figure 6

Diagnostic tests 